\title{
A Constant Factor Approximation for the Single Sink Edge Installation Problem*
}

\author{
Sudipto Guha ${ }^{\dagger} \quad$ Adam Meyerson ${ }^{\ddagger} \quad$ Kamesh Munagala ${ }^{\S}$
}

\begin{abstract}
We present the first constant approximation to the single sink buy-at-bulk network design problem, where we have to design a network by buying pipes of different costs and capacities per unit length to route demands at a set of sources to a single sink. The distances in the underlying network form a metric. This result improves the previous bound of $O(\log |R|)$, where $R$ is the set of sources. We also present a better constant approximation to the related Access Network Design problem. Our algorithms are randomized and combinatorial. As a subroutine in our algorithm, we use an interesting variant of facility location with lower bounds on the amount of demand an open facility needs to serve. We call this variant load balanced facility location, and present a constant factor approximation for it, while relaxing the lower bounds by a constant factor.
\end{abstract}

${ }^{*}$ This paper combines the work in two conference papers $[12,13]$, which appeared in the 41st IEEE Symposium on the Foundations of Computer Science, 2000 and the 33rd ACM Symposium on Theory of Computing, 2001 respectively.

${ }^{\dagger}$ Department of Computer and Information Sciences, University of Pennsylvania. Email: sudipto@cis.upenn.edu. Research supported by an NSF CAREER award and a Sloan Foundation Fellowship.

${ }^{\ddagger}$ Department of Computer Science, University of California, Los Angeles CA 90095-1596. Email: awm@cs.ucla.edu

${ }^{\S}$ Department of Computer Science, Duke University, Durham NC 27708. Email: kamesh@cs.duke.edu. Research supported by NSF via a CAREER award and grant CNS-0540347. 


\section{Introduction}

Network design problems require laying cables on an underlying metric in order to connect a set of demand points. The network must support each demand point operating at a known peak (or average) rate, and we would like the cheapest possible network supporting these demands. In a metric scenario (which is standard), if the cost of cables is linear in the amount of bandwidth they provide, this problem is polynomial-time solvable using multicommodity flow techniques. However, in several real applications the costs of cables obey economies of scale; the cost-per-unit-bandwidth is less for a high-capacity cable. Similar problems arise outside the data networks community, e.g., in location theory where we consider transporting products for sale where the cost is a concave function of the amount of demand transported. The concavity also may arise implicitly, e.g., in clustering data where we are willing to tolerate a less dense cluster if it contains a larger number of points.

The problem of buy-at-bulk network design, with a single sink node to which all the demand has to be routed, was first introduced by Salman et al in [21]. They gave a $O(\min \{\log n, \log D\})$ approximation, where the number of nodes is $n$ and $D$ is the maximum demand. They also showed that the problem is HP-hard. Awerbuch and Azar [3] which gave an $O\left(\log ^{2} n\right)$ approximation for the multiple sink case, where different demand points may communicate with different sinks. This result may be improved to $O(\log n)$ using subsequent results of $[4,5,9]$ on approximation of metrics using trees. Andrews and Zhang [2] considered the Access Network Design problem, where all demands need to connect to a single conceptual entity, the "core" of the network which represents the internet backbone, a set of file servers, or the factories where a product is produced. Even though there may exist multiple sinks, the sinks are symmetric in that demand points do not care to which sink they are connected. The access network design problem is a special case of single sink buy at bulk problem with the added twist that cables have to be utilized up-to a minimum capacity.

All of the above problems can be termed as an uniform variant, in the sense that any cable type can be used between any pair of vertices. For the more general non-uniform problem where cable types may have limited availability (but the network still has a single sink) Meyerson et al provided an $O(\log n)$ approximation in [19].

In this paper we focus on the uniform single sink case. Our main contributions are:

- Our algorithm provides the first constant factor approximation for the single-sink buy-at-bulk problem. This subsumes the Access network design problem [2] as well. The approximation ratio we obtain for the buy-at-bulk problem is 292 .

- We provide a Structure Theorem that allows us to identify the key regions in the concave function. This allows one to write an LP with $O(1)$ integrality gap. All subsequent analysis of this problem showing a $O(1)$ approximation (see below and Section 7 ) depend on this theorem as well.

- We define and provide the first constant factor bicriteria approximation algorithm for a natural variant of facility location, the Load Balanced Facility Location problem where there is a lower bound specified on the demand an open facility needs to serve. This problem arises in several clustering scenarios as well [14].

We provide randomized combinatorial algorithms, but they can be derandomized using standard techniques. The paper [12] contained several other results on multi-level network design problems which were based on similar techniques. We omit their discussion in the interest of keeping the presentation focused and simple. 
Solution Technique: There are several important novel ideas in our solution methodology. First, the optimal solution could use many cables with slightly differing costs in succession, obtaining marginal benefit from each. Such a solution is hard to characterize. We show that to a constant factor approximation, we can replace such similar cable types with the same cable type. This leads to a solution with simpler layered characterization (Structure Theorem). In particular, we show that a near-optimal solution is composed of alternating layers of Steiner and shortest path forests, each layer using only a single type of cable. The next layer gets used only when sufficient demand has been accumulated at the roots of the previous layer to make the next cable type cost-effective. Such a solution effectively hierarchically aggregates demand in order to exploit the economies of scale in cable costs.

The challenge now is to approximate the layered solution. We show that the shortest path instance corresponds to a variant of the well-studied facility location problem, where a certain minimum demand (corresponding to making the next cable type feasible) must be collected at the facilities. We present a constant factor approximation for this variant, which we term Load-balanced facility location. This is an independent contribution of this work. We present a simple constant factor approximation for the Steiner forest variant as well. We finally show that an iterative bottomup aggregation of demand using these Steiner and shortest path forests yields a constant factor approximation.

Independent Results: Independent of this work, Karger and Minkoff [16] defined the load balanced facility location problem as a subroutine for solving the single commodity Rent-or-Buy problem. They obtained the same algorithm as the one we present in Section 6 . We note that the single commodity Rent-or-Buy problem is a special case of the single sink buy-at-bulk problem considered in this paper.

Garg et al [10] obtained a $O(K)$ approximation (where $K$ is the number of different cable types) to the single sink buy-at-bulk problem by rounding the natural LP formulation. Our paper improves the approximation ratio via a fully combinatorial algorithm. Since the natural LP relaxations for the Steiner forest and shortest path problems have constant integrality gap, it is natural to expect that an LP based on the structure theorem should have $O(1)$ integrality gap as well, and we provide this LP for completeness. However, the authors of [10] observe that the structure theorem in our paper can be used on their stronger, natural LP, as well. This connection has also been made explicit by Talwar [23] subsequently.

Organization of the Paper: In Section 2, we state the single sink buy-at-bulk problem formally, and discuss the structural properties of the optimal solution. In Section 3.2, we discuss a scaling idea to remove similar pipe types, and show how it improves the structure of the optimum solution. We then present the HiERARCHY algorithm in Section 4 and show a constant approximation ratio. We show in Section 5 how to improve the approximation ratio for Access Network Design. We present the algorithm for load balanced facility location in Section 6, and conclude by surveying results that appeared subsequent to the publication of a preliminary version of this paper [13].

\section{Definitions and Preliminaries}

The Single Sink Buy at Bulk problem as defined in [21]: Given a graph $G(V, E)$ with a distance (length) function $c_{e}$ on the edges, the goal is to construct a network routing a set $S_{1} \subseteq V$ of demand nodes to a single sink $s$. We are given $K$ types of connections (pipes) where pipe type $i$ has a fixed cost $\sigma_{i}$ per unit length and a capacity $u_{i}$. Each demand node $v \in S_{1}$ needs to transport some amount of demand $d_{v}$ to the sink. The objective is to optimize the cost of buying pipes along the 
edges to route all demands to the sink. We are allowed to buy multiple copies of a pipe along the same link. The above can be termed as a capacitated version, we will use an alternate incremental cost formulation of the above problem, which easily models arbitrary piecewise linear concave costs.

Definition 2.1 In the single sink buy at bulk problem we are given a set of pipes, where pipe type $i$ has fixed and incremental costs $\sigma_{i}, \delta_{i}$ respectively. If we transport $d$ units of demand along a path of length $L$ using pipe type $i$, we will pay a total of $L\left(\sigma_{i}+\delta_{i} d\right)$. The goal is to construct a network routing a set $S_{1} \subseteq V$ of demand nodes to a single sink $s$, while minimizing the cost of the network.

Let $f_{i}(Y)=\sigma_{i}+\delta_{i} Y$ to be the per-unit-distance cost of routing demand $Y$ along a pipe of type $i$. Define $h(Y)=\min _{i} f_{i}(Y)$. The function $h()$ is piecewise linear and concave. Note that a pipe type which does not affect $h($ ) (that is, do not define the envelope) will not affect the solution at all. Therefore, if we only focus on the pipe types which are useful, and number the pipes in decreasing order of $\sigma_{i}$ we observe that $\delta_{1}>\delta_{2}>\cdots>\delta_{K}$. In this formulation the "capacity" of pipe $k$ is $u_{i}=\sigma_{i} / \delta_{i}$.

It is not hard to see that a solution under this incremental cost formulation costs at least as much as the same solution under the capacitated model, and at most twice as much as the solution under the capacitated model. Furthermore,

Lemma 2.1 [2] In the incremental cost model the optimum solution naturally defines a tree.

The above is straightforward since the costs are sub-additive under the above assumptions of $\left\{\sigma_{i}\right\},\left\{\delta_{i}\right\}$; after the links in the optimum are bought and the fixed cost paid, the entire demand from each node can be routed along the path with the lowest incremental cost (ties broken arbitrarily) from each node to the sink. This would define a tree, and we can eliminate the edges we do not use. Note that as a consequence, every node would use an unique pipe type for its outgoing flow. Also due to the subadditivity property of the costs, along every flow path the pipe types will increase in number.

The authors of [2] also introduced the following problem:

Definition 2.2 The Access Network Design problem is defined as follows: It is the same as the single sink buy at bulk problem with the following added restrictions ( $c=1 / 2$ is used in [2])

1. For $2 \leq k \leq K$, if $d<c \frac{\sigma_{k}}{\delta_{k}}$, then $d \delta_{k-1}+\sigma_{k-1}<d \delta_{k}+\sigma_{k}$.

2. The smallest demand looks like the smallest pipe capacity, or more precisely, $\delta_{1}>c \sigma_{1}$.

3. $\sum_{\kappa<k} \sigma_{\kappa}=O\left(\sigma_{k}\right)$.

As mentioned earlier, our solutions for the above problems will use solutions to the following variant of the Facility Location problem:

Definition 2.3 The Load Balanced Facility Location problem is defined as: We are given a network $G(V, E)$ with a distance function $c(\cdot)$ on the edges and a set of demand points, with demands $d_{j}$. The cost of opening a facility at location $i$ is $f_{i}$. In addition, there is a lower bound of $L_{i}$ on the demand a facility opened at $i$ must satisfy. We are required to open facilities and allocate the demands to the open facilities so that an open facility at $i$ has at least $L_{i}$ demand routed to it. The cost of our solution is the sum of the distances traveled by the demands and the cost of the open facilities. The goal is to minimize this cost. 


\section{Single Sink Buy at Bulk}

\section{$3.1 \quad$ Roadmap}

Intuition: Assume that we only consider the pipe types which are not dominated by others. Thus the cost per unit length is a piecewise concave function of the demand. Observe that as we increase demand along an edge, there are break-points at which it becomes cheaper to use the next larger pipe type. Let $g_{k}$ be the demand for which it becomes cheaper to use a pipe of type $k+1$ compared to a pipe of type $k$. Suppose that we are in a scenario $0=g_{0}<u_{1}<g_{1}<u_{2}<g_{2}<\cdots<u_{K}<g_{K}=\infty$ (we will show how to achieve a similar scenario later).

Observe now that if the demand amount is in the range $\left[g_{i-1}, u_{i}\right]$, we can ignore the incremental cost with a factor 2 loss in cost, and the cost of the edge will just be $\sigma_{i}$ times the length of the edge, independent of the demand. If on the other hand, the demand is in the range $\left[u_{i}, g_{i}\right]$, we can ignore the fixed cost with a factor 2 loss in cost, and the cost of the edge per unit length is $\delta_{i}$ times the demand. This implies that the optimum solution can be converted with a factor 2 loss in cost to a layered solution. Layer $i$ has a Steiner forest using pipes of type $i$ followed by a forest of shortest path trees using pipes of the same type. Each pipe in the Steiner forest has at least $g_{i-1}$ demand and each pipe in the shortest path forest has at least $u_{i}$ amount of demand. The shortest path forest should ensure that we collect at least a demand of $g_{i}$, such that we can use the Steiner forest corresponding to a larger pipe type. This gives us a clustering problem where each cluster is supposed to have a minimum number of (weighted by demand) points - this is the reason for using the load balanced facility location problem.

For completeness, we first define the facility location problem [22]:

Definition 1 (Facility Location) We are given a set of demands D. Let $d_{j}$ be the demand at $j \in D$. We are given a set of feasible locations $F$, where $\phi_{i}$ is the cost of opening a facility at location $i \in F$. The points $D$ and $F$ are embedded in a metric space where $c_{i j}$ is the distance between points $i$ and $j$. The goal is to open a subset of facilities $X \subseteq F$ and connect each demand $j \in D$ to the closest open facility $q(j) \in X$, so that the total cost of the open facilities, $\sum_{i \in X} \phi_{i}$ plus the sum of the routing cost, $\sum_{j \in D} c_{q(j) j} d_{j}$ is minimized. Let $\rho_{f}$ denote the best approximation ratio for the facility location problem. This is 1.52 due to [18].

The Load Balanced Facility Location problem has an added constraint: Each $i \in F$ has a lower bound $L_{i}$ on the demand it needs to serve if opened. The solution $X$ constructed must satisfy that for every $i \in X$, at least $L_{i}$ demand is routed to $i$ in the solution. In Section 6 we prove the following:

Theorem 3.1 We can compute a solution for load balanced facility location whose cost is $2 \rho_{f}$ times that of the optimal solution, such that our solution relaxes the lower bounds by a factor of $1 / 3$, so that for $i \in X$, at least $L_{i} / 3$ demand is routed to $i$.

However, if we apply the above directly and compare ourselves with the optimal solution that satisfies the above mentioned structural properties. the analysis does not immediately go through. So we use the idea that we will only use a larger pipe type when it is significantly, i.e., by a constant factor, cheaper. This will allow us to set up a geometric series that accounts for the cost of the shortest path forests. But to bound the cost of the Steiner forests we will need a different idea, namely we restrict ourselves to pipes where the $\sigma_{i}$ decrease by a constant factor as well. But this now implies that we should show that even after ruling out pipes according to the above two ideas, there is a feasible solution which is not too expensive. This is the structure theorem we prove. 
There is however one remaining issue regarding how to correlate the costs of the different layers - we introduce a novel strategy where the entire demand of a layer is sent to random node in $S_{1}$. Note that it is important for our analysis that the demands be sent to $S_{1}$, because the structured solution we derive from modifying the optimum solution is defined with these demand nodes as the ground truth.

In the remainder of the section, we first describe the structured feasible solution in Section 3.2. We then present the algorithm in Section 4.

\subsection{Constructing a Layered Solution}

We now formalize the intuition described above to obtain a layered solution with cost close to the optimal cost. Our algorithm will progressively construct partial solutions using each pipe type in turn. In order to bound the total cost, we must guarantee that pipes are very different from one another in terms of fixed and incremental costs.

Definition 3.1 Define a set of pipe types to be good if for some $\alpha \in(0,1 / 2)$ we have:

1. For any $k<K$, we have $\sigma_{k}<\alpha \sigma_{k+1}$.

2. For any $k<K$, we have $\alpha \delta_{k}>\delta_{k+1}$.

We need to prove that we can guarantee these conditions without increasing the cost of the optimum solution by too much.

Lemma 3.1 There exists a set of good pipes and a solution that uses only these types, such that the cost of this solution is at most $1 / \alpha$ times the cost of the original optimum solution.

Proof: We first eliminate pipes in order to guarantee that among the remaining pipes we have $\sigma_{k}<\alpha \sigma_{k+1}$ while increasing the fixed cost of the optimum solution by at most $1 / \alpha$. The incremental cost of the optimum solution can only decrease during this phase.

We find the largest pipe $k$ such that $\sigma_{k} \geq \alpha \sigma_{k+1}$. We eliminate this pipe, replacing it in the optimum solution with pipe $k+1$. We renumber the pipes and repeat. Notice that if at some point some pipe type is replaced by pipes of type $k$, then we will always keep pipes of type $k$ in the final solution (since every pipe type higher than $k$ has at least $\alpha$ higher fixed cost). When this finishes, we will have the desired property. The original optimum solution with pipe replacements has fixed cost at most $1 / \alpha$ larger since any pipe which was replaced was replaced by a pipe with at most $1 / \alpha$ bigger fixed cost. The incremental cost can only decrease, since higher fixed cost implies smaller incremental cost.

We now eliminate pipes in reverse order, where an eliminated pipe is replaced by a pipe with larger incremental cost, to guarantee that among the remaining pipes we have $\alpha \delta_{k}>\delta_{k+1}$ while increasing the incremental cost of the optimum solution by at most $1 / \alpha$. The fixed cost of the optimum solution can only decrease.

Combining these two phases gives the solution claimed by the lemma.

Definition 3.2 Assuming that we have a good set of pipes, define $b_{k}$ to be such that $f_{k+1}\left(b_{k}\right)=$ $2 \alpha f_{k}\left(b_{k}\right)$. In essence, $b_{k}$ is sufficient demand that it becomes considerably cheaper to use a pipe of type $k+1$ rather than a pipe of type $k$. 
Lemma 3.2 For all $k, u_{k} \leq b_{k} \leq u_{k+1}$.

Proof: From the definition of $b_{k}$, we can write: $\sigma_{k+1}+\delta_{k+1} b_{k}=2 \alpha\left(\sigma_{k}+\delta_{k} b_{k}\right)$.

Solving this equation for $b_{k}$ yields:

$$
b_{k}=\frac{\sigma_{k+1}-2 \alpha \sigma_{k}}{2 \alpha \delta_{k}-\delta_{k+1}} \leq \frac{\sigma_{k+1}}{2 \alpha \delta_{k}-\delta_{k+1}} \leq \frac{\sigma_{k+1}}{\delta_{k+1}}=u_{k+1}
$$

The above shows $b_{k} \leq u_{k+1}$, to see the other bound observe that when we have $b_{k}$ flow, it is cheaper to use a pipe of type $k+1$ rather than a pipe of type $k$. It follows that $\sigma_{k+1}+\delta_{k+1} b_{k}<$ $\sigma_{k}+\delta_{k} b_{k}$. Solving this for $b_{k}$, we can see that

$$
b_{k}>\frac{\sigma_{k+1}-\sigma_{k}}{\delta_{k}-\delta_{k+1}}
$$

Since $\alpha<1 / 2$, it follows that $\sigma_{k+1}>2 \sigma_{k}$ and we can conclude that $b_{k}>u_{k}$.

Lemma 3.3 For all $k$ and any demand $D \geq b_{k}, f_{k+1}(D) \leq 2 \alpha f_{k}(D)$.

Proof: Suppose $D=b_{k}+x$ for some $x \geq 0$. Then, $f_{k+1}(D)=\sigma_{k+1}+\delta_{k+1}\left(b_{k}+x\right)=2 \alpha\left(\sigma_{k}+\right.$ $\left.\delta_{k} b_{k}\right)+\delta_{k+1} x$. Noting that $\delta_{k+1} \leq \alpha \delta_{k}$, it immediately follows that $f_{k+1}(D) \leq 2 \alpha f_{k}(D)$.

Lemma 3.4 For all $k$ and any demand $D \leq u_{k}, f_{k+1}(D) \geq f_{k}(D)$.

Proof: Note that $f_{k+1}(D), f_{k}(D)$ are nondecreasing linear functions in $D$ and further, $\delta_{k+1} \leq \delta_{k}$. Therefore, to prove the lemma, it suffices to observe that $f_{k+1}\left(u_{k}\right) \geq f_{k}\left(u_{k}\right)$.

But $f_{k}\left(u_{k}\right)=\sigma_{k}+\delta_{k} \frac{\sigma_{k}}{\delta_{k}}=2 \sigma_{k}<\sigma_{k} / \alpha \leq \sigma_{k+1} \leq f_{k+1}\left(u_{k}\right)$. Note that we require $\alpha \in\left(0, \frac{1}{2}\right)$.

We now show that there exists a structured near-optimum solution. Subsequently we will search for solutions which obey this structure and are within constant factor of the best structured solution.

Theorem 3.2 (Structure Theorem) There exists a tree solution that uses pipes of type $k$ on a link iff the demand $x$ on the link satisfies $x \in\left[b_{k-1}, b_{k}\right)$. Further, the tree routes all demand which entered a node using pipe $k$ out of that node using a pipe of type $k$ or $k+1$. This solution pays at most $\frac{1}{2 \alpha^{2}}$ times the optimum solution.

Proof: First, as noted in Section 2, the optimal solution defines a tree due to the sub-additive nature of the costs. Further, as noted there, the incremental cost model forces this solution to use only one pipe type per edge. Both these properties will be preserved by the transformations described below.

We first modify the solution to only use the set of good pipes (according to Lemma 3.1). Therefore we need to show that transformations in the the rest of the proof increases the cost of the solution by at most a factor of $\frac{1}{2 \alpha}$.

Consider any edge where $x$ units of flow is routed by the optimum solution. Let $k_{0}=\operatorname{argmin}_{i} f_{i}(x)$. This is the pipe type used by the optimum solution.

Suppose a pipe has flow $b_{k-1} \leq x<u_{k}$. We know by (repeated) application of Lemmas 3.3 and 3.2 that a pipe of type $k$ would reduce the cost compared to any smaller pipe type. Likewise, by 
(repeated) application of lemmas 3.4 and 3.2 we can conclude that a pipe of type $k$ would reduce the cost compared to any larger pipe type. Thus $k=k_{0}$.

Therefore, what remains to be shown is that if $u_{k} \leq x<b_{k}$ and we use a pipe of type $k$, then our cost does not increase significantly. First, due to the discussion above, since $b_{k} \leq u_{k+1}$, we know that it is cheaper to use pipe type $k+1$ compared to any larger pipe type. Thus $k_{0}=k$ or $k_{0}=k+1$ and we need to compare $f_{k}(x)$ and $f_{k+1}(x)$ only. Now by Definition 3.2, and the fact that $f_{k}(x)$ and $f_{k+1}(x)$ are linear non-decreasing with $\delta_{k+1} \leq \delta_{k}$; it is immediate that $2 \alpha f_{k}(x) \geq f_{k+1}(x)$.

Thus if we modify the optimum solution (already restricted to good pipes) to use pipe type $k$ in the range $\left[b_{k-1}, b_{k}\right)$, then the cost of the solution goes up by at most a factor of $1 /(2 \alpha)$; combined with Lemma 3.1 the total cost is at most $1 /\left(2 \alpha^{2}\right)$ times the optimal cost. To achieve the second part of the lemma, we observe that since we are considering a tree solution, the flow does not decrease as we proceed toward the root. We simply introduce dummy nodes or pipes of length 0 , if the largest incoming pipe type is $k$ and the outgoing pipe type is larger than $k+1$.

An LP Formulation: We can encode the structural observation above into an integer program formulation. We modify the graph to include $K$ self-loops of length 0 at every vertex, in order to accomodate pipe types required by the Structure Theorem.

Denote by $x_{v e k}$ whether the demand at node $v$ uses a pipe of type $k$ on edge $e$. By $y_{e k}$ we denote whether there exists a pipe of type $k$ on edge $e$. The integer program can then be formulated as follows. Here $\operatorname{In}(v)$ denotes the set of edges coming into on node $v$, and $\operatorname{Out}(v)$ the set going out of $v$. Recall that $c_{e}$ is the length of edge $e$, and $d_{v}$ is the demand at node $v \in S_{1}$.

$$
\begin{array}{rlrl}
\text { Minimize } & \sum_{e \in E} c_{e}\left(\sum_{k} \sigma_{k} \cdot y_{e k}+\sum_{e} \sum_{v \in S_{1}} \sum_{k} \delta_{k} \cdot d_{v} \cdot x_{v e k}\right) \\
\sum_{e \in \operatorname{In}(w)} x_{v e k} & =\sum_{e \in \operatorname{Out}(w)}\left(x_{v e k}+x_{v e k+1}\right) & & \forall v \in S_{1}, w \in V \backslash\{s\}, k \\
x_{v e k} & \leq y_{e k} & & \forall v \in S_{1}, e \in E, k \\
\sum_{e \in \operatorname{Out}_{(v)} x_{v e 1}}=1 & & \forall v \in S_{1} \\
x_{v e k}, y_{e k} & \in\{0,1\} &
\end{array}
$$

The LP is obtained by relaxing the final integrality constraints. It can be shown using Theorem 4.1 that if the LP is written on the set of good pipe types, it has an $O(1)$ integrality gap. Direct LP rounding techniques exist as well [10, 23]. Since the LP is not the main focus of the paper, we omit a proof of the integrality gap.

\section{The Hierarchy Algorithm}

We will now present the HIERARCHY algorithm for single sink buy-at-bulk based on the structural observations we made above. The scaling idea from the previous section measures that we can compare the cost of our solution in each layer with the respective costs of the optimum solution. The algorithm is presented below: 


\section{Algorithm HIERARCHY}

- Let $s$ denote the sink node and $S_{1}$ the set of original demand nodes. Assume $s \in S_{1}$. Let the demand of $v \in S_{1}-\{s\}$ be denoted by $d_{v}$.

- The algorithm proceeds in phases. In phase $i$ we will use pipes of type $i$ only. Let $D_{i}(v)$ denote the demand of a node $v$ at the start of the phase. Since the algorithm is randomized, this is a random variable. Let $S_{i}$ be the set of non-zero demand points (and $s$ ) we have at this stage.

(i) Steiner Trees: Construct an approximately optimal Steiner tree on $S_{i}$. Root this tree at $s$. Transport the demands from $S_{i}$ upwards along the tree. If on any edge, the amount of demand is larger than $u_{i}$, we "cut" the tree at that edge. This gives us a forest on $S_{i}$ where each edge has at most $u_{i}$ demand through it.

(ii) Consolidate A: Consider any subtree $t$ in the above forest which does not contain $s$. Let the set of nonzero demand nodes in $t$ be $S_{i}^{A}(t)$. Pick a node $y$ at random from $S_{i}^{A}(t)$ in proportion to its demand $D_{i}(y)$. For all nodes in $S_{i}^{A}(t)$, we send their demand (which are currently located at the root of $t$ ) to $z$ using pipes of type $i$. Let $A_{i}$ be the set of nodes $y$ chosen corresponding to different $t$. Denote the demand of a node $v$ immediately after this step to be $D_{i}^{A}(v)$.

(iii) Shortest Path Trees: Approximately solve a load balanced facility location instance on $S_{1}$ with the facility lower bound $b_{i}$ on all nodes (and no facility costs). If there does not exist $b_{i}$ total demand, then we instead route directly to the sink. We get a forest of shortest path trees. We route our current demands along these trees to their roots. Note that we solve the load balanced facility location problem on the $S_{1}$ nodes and not on the $S_{i}^{A}(t)$ nodes.

(iv) Consolidate B: Consider any facility $p$ opened in the above forest of shortest path trees. Some set of nodes from $S_{1}$ were assigned to $p$, denoted by $S_{i}^{B}(p)$, and their (original) total demand is at least $b_{i} / 3$. We choose a node $z$ at random from $S_{i}^{B}(p)$ with probability proportional to $d_{z}$. For all $y \in S_{i}^{B}(p) \cap A_{i}$, we send their demand (which is currently at node $p$ ) to node $z$ using pipes of type $i$. Let $S_{i+1}$ be the set of nodes $z$ that are chosen corresponding to the different facilities $p$. Note that the only nodes currently having non-zero demands are nodes in $S_{i+1}$.

Our solution will route the demands through the forests of increasing pipe types. This solution need not be a tree, but can easily be converted to one of no greater cost.

Let $\rho_{s}$ and $\rho_{f}$ denote the best approximation ratios for the Steiner tree and facility location problems respectively. Note that $\rho_{s}=1.55$ due to [20] and $\rho_{f}=1.52$ due to [18]. Note that for the "Shortest Path tree" part, we use Theorem 3.1 to obtain a $2 \rho_{f}$ approximation that routes at least $b_{i} / 3$ demand to each open facility. For the "Steiner Trees" part, we use the $\rho_{s}$ approximation.

\subsection{Analysis}

Let $\Gamma$ be the structured optimal solution constructed in Theorem 3.2. We define $C_{i}^{*}$ to be the total cost of $\Gamma$ using pipes of type $i$. The total cost of the structured optimal solution is therefore $\sum_{i=1}^{K} C_{i}^{*}=C^{*}$.

Let $T_{i}^{I}$ be the incremental cost of the Steiner Tree at layer $i$ and $T_{i}^{F}$ be its fixed cost. The total cost of the Steiner Tree at layer $i$ is $T_{i}=T_{i}^{I}+T_{i}^{F}$.

Let $P_{i}^{I}$ be the incremental cost of the shortest path tree at layer $i$ and $P_{i}^{F}$ be its fixed cost. The total cost of the shortest path tree at layer $i$ is $P_{i}=P_{i}^{I}+P_{i}^{F}$.

Let $N_{i}$ be the total cost of the consolidation steps for layer $i$. The total cost of our solution is therefore $\sum_{i}\left(T_{i}+P_{i}+N_{i}\right)$. 
Lemma 4.1 For all $i, v$, we have $\mathbf{E}\left[D_{i}(v)\right]=d_{v}$ and $\mathbf{E}\left[D_{i}^{A}(v)\right]=d_{v}$, that is the expected demand at any node after any of the consolidation steps is the original demand of the node.

Proof: We will prove this by induction on the steps $i$. Suppose that the statement is true at some step. We will show that it is true at the next step. There are two cases to consider; either we performed a Steiner Tree step or a Shortest Path Tree step.

Note $D_{1}(v)=d_{v}$. For the ease of notation, define $D_{0}^{A}(v)=d_{v}$ for all nodes $v$. These two equations define the base case.

Suppose we have just performed a Steiner Tree step. By the induction hypothesis we know $\mathbf{E}\left[D_{i}(v)\right]=d_{v}$. The node $v$ is a part of some tree $t$ with total demand $D_{t}$. We then choose a node for consolidation and the probability that we choose node $v$ is $D_{i}(v) / D_{t}$. If we choose $v$, demand $D_{t}$ will be placed there; otherwise the demand is 0 . Thus the expected amount of demand at $v$, conditioned on the previous $i-1$ steps is $D_{i}(v)$. If we now remove the conditioning, $\mathbf{E}\left[D_{i}^{A}(v)\right]=\mathbf{E}\left[D_{i}(v)\right]=d_{v}$ as desired.

Suppose we have just performed a Shortest Path Tree step. Note that we used the $S_{1}$ nodes for the load balanced facility location construction in this step. The probability we consolidate to $v$ is $d_{v} / D(p, i)$ where $v$ is assigned to $p$ in this stage and the total demand assigned to $p$ is $D(p, i)$. Note that

$$
D(p, i)=\sum_{u \in S_{i}^{B}(p)} d_{u}
$$

The demand of $v$ is $D_{i+1}(v)$ after this step. Note that $v$ collects the demands of the nodes in set $A_{i} \cap S_{i}^{B}(p)$; further the demand of a node $u$ in this intersection is $D_{i}^{A}(u)$.

Conditioned on the previous steps, the expected value of $D_{i+1}(v)$ is $\frac{d_{v}}{D(p, i)} \sum_{u \in S_{i}^{B}(p)} D_{i}^{A}(u)$. Now if we remove the conditioning,

$$
\mathbf{E}\left[D_{i+1}(v)\right]=\frac{d_{v}}{D(p, i)} \mathbf{E}\left[\sum_{u \in S_{i}^{B}(p)} D_{i}^{A}(u)\right]=\frac{d_{v}}{D(p, i)} \sum_{u \in S_{i}^{B}(p)} \mathbf{E}\left[D_{i}^{A}(u)\right]=\frac{d_{v}}{D(p, i)} \sum_{u \in S_{i}^{B}(p)} d_{u}=d_{v}
$$

This proves the inductive case.

Lemma $4.2 \mathbf{E}\left[N_{i}\right] \leq T_{i}+P_{i}$.

Proof: The proof essentially follows by observing that the cost is a concave function of demand, and the consolidation process picks a random node in proportion to the demand sent to the root.

This is obvious for the shortest path trees - let the nodes that belonging to a tree rooted at facility $p$ be $v_{1}, \ldots, v_{r}$ with distances $\ell_{1}, \ldots, \ell_{r}$ from node $p$. Then the expected consolidation cost is

$$
\sum_{p} \sum_{a=1}^{r}\left(d_{v_{a}} / D\right) f_{i}(D) \ell_{a} \leq \sum_{p} \sum_{a=1}^{r} f_{i}\left(d_{v_{a}}\right) \ell_{a}=P_{i}
$$

The inequality follows since $f_{i}$ is a concave function.

For the other step, consider the consolidation process to recursively choose from the root a subtree with probability proportional to the total demand in the subtree. We just saw the proof for a 1 level tree that the consolidation cost is less than the cost of sending the flows to the root. This argument is now repeated in each of the subtrees.

Lemma $4.3 T_{i}^{I} \leq T_{i}^{F}$ and $P_{i}^{F} \leq P_{i}^{I}$. 
Proof: Since we cut the tree at any edge with more than $u_{k}$ demand along it, we guarantee that the fixed cost paid on any edge we actually use exceeds the incremental cost.

By the same argument, the Steiner Tree stage guarantees at least $u_{k}$ demand or zero everywhere. For the shortest path tree, if an edge has zero demand flowing on it, we will pay zero for that edge. Otherwise there is at least $u_{k}$ demand on the edge and we pay an incremental cost which exceeds the fixed cost for the shortest path trees.

Lemma 4.4 $\mathbf{E}\left[P_{i}^{I}\right] \leq 2 \rho_{f} \sum_{j=1}^{j=i} \alpha^{i-j} C_{j}^{*}$.

Proof: Suppose the demands at the sources were those from $S_{1}$. Then one possible solution would be $\Gamma$ itself until pipes of type $i+1$ were used. We know that $\Gamma$ must gather the desired $b_{i}$ flow before using pipes of type $i+1$. Since we will always pay the incremental cost $\delta_{i}$, and the incremental costs scale by $\alpha$, we can guarantee a total cost of at most $\sum_{j=1}^{j=i} \alpha^{i-j} C_{j}^{*}$ for this solution. Our actual demand at each node has expected value equal to the original demand, so the expected value of a feasible solution for $P_{i}^{I}$ is bounded as above. The extra factor is due to the approximation of the load balanced facility location problem as stated in Theorem 3.1.

Lemma 4.5 $\operatorname{Pr}\left[D_{i}(v) \neq 0\right] \leq 3 d_{v} / b_{i-1}$.

Proof: Let $v$ be the chosen node (denoted by $z$ in Step (iv) of the algorithm) corresponding to some $p$. We obtain the nodes $S_{i}$ by solving an instance of the load balanced facility location problem on $S_{1}$ with lower bounds $b_{i-1}$. In this solution, each node in $S_{i}$ except $s$ has demand at least $b_{i-1} / 3$. In other words $\sum_{u \in S_{i}^{B}(p)} d_{u} \geq b_{i-1} / 3$.

Now note that $v$ is chosen independent of $\left\{D_{i-1}(u)\right\}$ - and this is the reason why $S_{1}$ is used and not $S_{i}$. Note that at any node $u$ in $S_{1}$, the currently accumulated before the shortest path tree step is $D_{i-1}^{A}(u)$. We therefore have:

$$
\mathbf{E}\left[D_{i}(v) \mid D_{i}(v)>0\right]=\sum_{u \in S_{i}^{B}(p)} \mathbf{E}\left[D_{i-1}^{A}(u)\right]=\sum_{u \in S_{i}^{B}(p)} d_{u} \geq b_{i-1} / 3
$$

Now combined with the fact that $\mathbf{E}\left[D_{i}(v)\right]=d_{v}$, the lemma follows.

Lemma 4.6 Recall $\rho_{s}$ be the approximation ratio the Steiner tree approximation algorithm used, then $\mathbf{E}\left[T_{i}^{F}\right] \leq \rho_{s}\left(\sum_{j=i}^{j=K} \alpha^{j-i} C_{j}^{*}+\sum_{j=1}^{j=i-1} 3(2 \alpha)^{i-j} C_{j}^{*}\right)$.

Proof: To bound the cost of $T_{i}^{F}$, we will show that there exists a Steiner tree $\Gamma_{i}$ which connects all $S_{i}$ with a low cost. Note that this Steiner tree will have an approximation ratio at most $\rho_{s}$ relative to the optimal Steiner tree on the set $S_{i}$.

The tree $\Gamma_{i}$ will be the tree corresponding to the structured solution $\Gamma$, where (1) all the pipes of type $j \geq i$ use pipes of type $i$ and (2) for the nodes $v$ with $D_{i}(v)>0$ the edges in the path to the root where any pipe of type $j<i$ is used, that corresponding edge now uses a pipe of type $i$.

The cost according to part (1) can be bounded by $\sum_{j=i}^{j=K} \alpha^{j-i} C_{j}^{*}$. This is because for any pipe of type $j \geq i$, we have $\sigma_{i} \leq \alpha^{j-i} \sigma_{j}$. Further, the fixed cost of using the pipe of type $j$ is less than its total cost and thus the above bound follows.

To bound the contribution of (2), we focus on the subtrees of $\Gamma$ which has total demand at most $b_{i-1}$ (and therefore use pipes of type $j<i$ ). Note that due to the introduction of zero length edges, these subtrees may share their (sub)roots - but they will be edge disjoint. For an edge $e$, 
let the demand flowing through it be $x_{e}<b_{i-1}$ and suppose that $\Gamma$ uses a pipe of type $j_{e}<i$ for this edge. Let the set of nodes in $\Gamma$ below $e$ be denoted by $\Gamma_{e}$. Let the length of $e$ be $\ell_{e}$.

Now the edge $e$ is used in $\Gamma_{i}$ if any $v$ in the subtree below it has $D_{i}(v)>0$. Therefore the probability $e$ is used is bounded above by $\sum_{v \in \Gamma_{e}} 3 d_{v} / b_{i-1}$ using Lemma 4.5 and the union bound. Thus we pay a cost

$$
\begin{aligned}
\sum_{v \in \Gamma_{e}} \frac{3 d_{v}}{b_{i-1}} \ell_{e} \sigma_{i} & =\frac{3 x_{e}}{b_{i-1}} \sigma_{i} \ell_{e} \leq \frac{3 x_{e}}{b_{i-1}} f_{i}\left(b_{i-1}\right) \\
& \leq \frac{3 x_{e}}{b_{i-1}}(2 \alpha)^{i-j_{e}} f_{j_{e}}\left(b_{i-1}\right) \leq 3(2 \alpha)^{i-j_{e}} f_{j_{e}}\left(\frac{x_{e}}{b_{i-1}} b_{i-1}\right)=3(2 \alpha)^{i-j_{e}} f_{j_{e}}\left(x_{e}\right)
\end{aligned}
$$

The second inequality follows from Lemma 3.3. Therefore if we sum the right hand side over all $j_{e}$, the contribution from edges in that level will be $3(2 \alpha)^{i-j_{e}} C_{j_{e}}^{*}$. Taking the contribution of both (1) and (2) together, the total cost of $\Gamma_{i}$ is at most

$$
\sum_{j=i}^{j=K} \alpha^{j-i} C_{j}^{*}+\sum_{j=1}^{j=i-1} 3(2 \alpha)^{i-j} C_{j}^{*}
$$

We can find a Steiner Tree of cost at most $\rho_{s}$ times the above cost, so the lemma follows.

Theorem 4.1 The HIERARCHY algorithm is a constant-approximation for the single-sink buy-atbulk problem.

Proof: By Lemmas 4.2 and 4.3, the total expected cost of our solution, $\sum_{i} \mathbf{E}\left[N_{i}+P_{i}+T_{i}\right]$, is bounded by $\sum_{i} 2\left(2 T_{i}^{F}+2 P_{i}^{I}\right)$. Using Lemmas 4.4 and 4.6 , we conclude that the expected cost of our solution is bounded by the following:

$$
4 \sum_{i}\left(\rho_{s} \sum_{j=i}^{j=K} \alpha^{j-i} C_{j}^{*}+\rho_{s} \sum_{j=1}^{j=i-1} 3(2 \alpha)^{i-j} C_{j}^{*}+2 \rho_{f} \sum_{j=1}^{j=i} \alpha^{i-j} C_{j}^{*}\right)
$$

By reversing orders of summation, we can bound this by:

$$
4\left(\frac{\rho_{s}}{1-\alpha}+\frac{6 \alpha \rho_{s}}{(1-2 \alpha)}+\frac{2 \rho_{f}}{1-\alpha}\right) C^{*}
$$

This is our approximation relative to the near-optimum structured solution. Using Theorem 3.2 allows us to bound our overall approximation ratio by:

$$
\left(\frac{4}{2 \alpha^{2}}\right)\left(\frac{\rho_{s}+2 \rho_{f}}{1-\alpha}+\frac{6 \alpha \rho_{s}}{1-2 \alpha}\right)
$$

The best known approximation ratio for Steiner trees is $\rho_{s}=1.55$ due to [20], and that for facility location is $\rho_{f}=1.52$ due to [18]. Setting $\alpha=1 / 3$ the above reduces to $54 \rho_{f}+135 \rho_{s}<292$ approximation for the incremental cost model. 


\section{Improved Approximation Algorithm for Access Network Design}

Andrews and Zhang [2] consider the case $c=1 / 2$ and show that the optimal solution can be converted with a constant factor loss into a layered solution of shortest path forests. They show that there exists a near-optimal (within a constant multiplier on the cost) solution which is a tree satisfying the following properties:

1. Each demand is routed through pipes of consecutive types, i.e. types $1,2, \ldots, \kappa .(\kappa \leq k)$.

2. For all pipe types $k$, any pipe of that type has at least $u_{k} / 2=\frac{\sigma_{k}}{2 \delta_{k}}$ amount of demand flowing through it.

This means that for Access Network Design, the optimal solution can be converted to a layered solution using shortest path forests of increasing pipe types.

We can improve the analysis of the above algorithm for Access Network Design. As shown in [12], for the Access Network Design we have a layered shortest path forest solution with a reduction in cost at each layer. We can prove the following theorem:

Theorem 5.1 There exists a solution to the Access Network Design problem in which we only use pipe types satisfying the condition $\phi_{i}=\frac{\delta_{i+1}}{\delta_{i}} \leq \alpha$, and in which any pipe of type $i$ has at least $u_{i} / 2$ amount of demand flowing through it. The fixed and incremental costs of this solution are each within $\frac{1}{\alpha}$ of the original optimum which used all pipe types and which had at least $u_{k} / 2$ demand in any pipe of type $k$.

Proof: Note that since we are using pipes of larger types in increasing layers, the incremental cost $\delta$ per unit of traffic keeps decreasing. In fact, we can make sure that $\delta$ goes down by a constant fraction $\alpha<1$ with a $\frac{1}{\alpha}$ increase in cost. The way we do this is the following:

Consider pipes of increasing types starting at type 1 . Let $\phi_{i}=\frac{\delta_{i+1}}{\delta_{i}}$. Let $k^{\prime}$ be the largest number such that $\prod_{i=1}^{k^{\prime}} \phi_{i} \geq \alpha$. We remove all pipe types $2, \ldots, k^{\prime}+1$ and use only pipe of type 1 instead of all these pipes. We next consider pipes starting at type $k^{\prime}+2$ and repeat this filtering process.

When the above is completed, we are left with a set of pipe types satisfying the following properties. For consecutive pipe types $i$ and $i+1, \frac{\delta_{i+1}}{\delta_{i}} \leq \alpha$. Finally, note that in this process, if a pipe of type $j$ is replaced by a pipe of type $i$, it must be the case that $\phi_{i}<\phi_{j}$, and $\delta_{i}<\frac{1}{\alpha} \delta_{j}$, so that the cost of using pipe $i$ is at most $\frac{1}{\alpha}$ times the cost of using pipe $j$.

Recall $\phi_{i}=\frac{\delta_{i+1}}{\delta_{i}}$. From above, we can assume with a loss of $\frac{1}{\alpha}$ in the approximation ratio that all $\phi_{i} \leq \alpha<1$. Our algorithm will lay pipes in increasing order of types.

Let $S_{i}$ denote the demand points at stage $i$. We maintain the invariant that every demand point has at least $u_{i} / 6$ demand. We solve the load balanced facility location instance on $S_{i}$ with lower bound $u_{i+1}$ (except on the sink $s$ ). We route the demands to the open facilities using pipes of type $i$. For every open facility, we choose one of the demand points sending demand to it at random in proportion to its demand, and route all the demand to this point using pipes of type $i+1$. Let $S_{i+1}$ be the final set of demand points to where we route the demands. Note that every demand point has at least $u_{i+1} / 6$ demand.

Let $P_{i}^{I}$ be the routing cost at stage $i$, and let $P_{i}^{F}$ be the fixed cost. Note that $P_{i}^{F} \leq 6 P_{i}^{I}$ because of the invariant on the demands.

We define $C_{i}^{*}$ to be the total incremental cost incurred by the optimal solution using pipes of type $i$. Note that the total cost of the optimal solution is $C^{*} \geq \sum_{i} C_{i}^{*}$. 
Lemma $5.1 \mathbf{E}\left[P_{i}^{I}\right] \leq 2 \rho_{f}(1+\alpha)\left(\sum_{j=1}^{j=i-1} \alpha^{i-j-1} C_{i}^{*}\right)$.

Proof: The routing cost that the optimum solution pays in routing the original demand points till stage $i$ using pipes of type $i$ is at most $\sum_{j=1}^{j=i-1} \alpha^{i-j-1} C_{i}^{*}$. This follows from [12] and from the analysis in Section 4. This is an instance of the load balanced facility location problem, and we apply Theorem 3.1 .

It is now easy to see the following.

Lemma $5.2 \mathbf{E}\left[\sum_{i}\left(P_{i}^{I}+P_{i}^{F}\right)\right] \leq 14 \rho_{f} \frac{1+\alpha}{1-\alpha} C^{*}$.

Note that we lost a factor of $\frac{1}{\alpha}$ up front in the routing cost because of scaling the pipe types. Our approximation ratio is therefore $\frac{1}{\alpha} 14 \rho_{f} \frac{1+\alpha}{1-\alpha}$. Setting $\alpha=1 / 3$ and $\rho_{f}=1.52$ this ratio is less than 128.

Theorem 5.2 We have a randomized 128 approximation for Access Network Design.

This approximation factor has been subsequently improved in [15].

\section{Load Balanced Facility Location}

Recall the definition of facility location and load balanced facility location from Section 3.1. The load balanced problem differs from standard facility location [22] in that we must route at least $L_{i}$ units of demand to each open facility $i$. Load Balanced Facility Location has direct applications; consider a franchise which must open stores to minimize the average distance from customer to store, but which must also guarantee a minimum number of customers to each store so the individual stores remain profitable. We present a constant approximation to this problem, losing a constant factor compared to the lower bound on demand. We can write an integer program for this problem.

$$
\begin{aligned}
\operatorname{Minimize} \sum_{i} \sum_{j} d_{j} c_{i j} x_{i j}+\sum_{i} \phi_{i} y_{i} \\
\sum_{i} x_{i j} \geq 1 \quad \forall j \\
x_{i j} \leq y_{i} \quad \forall i, j \\
\sum_{j} d_{j} x_{i j} \geq L_{i} y_{i} \quad \forall i \\
x_{i j}, y_{i} \in\{0,1\} \quad \forall i, j
\end{aligned}
$$

Clearly, the general version of this problem is NP-hard, as it reduces to classical facility location when the lower bounds are set to zero. In fact, this problem is NP-hard even if all facility costs are zero, all lower bounds are equal and all demands are unit.

Theorem 6.1 Suppose we are given a load balanced facility location instance with lower bound $L$ on all facility locations, and facility costs being zero. Deciding if a feasible solution of cost at most $C$ exists is NP-hard.

Proof: We reduce the decision version of the unweighted set cover problem to an instance of this problem as follows. The sets are the facilities. The elements are the demand points with unit demand. Suppose there are $n$ elements. In the facility location instance, we add edges of cost one between every element and all the sets it belongs to. 
Suppose we have to decide if a cover with $s>1$ sets exists. We add $s n$ demand points with unit demand, and connect them to all the sets (or facilities) with edges of length one. We set the lower bound on the facilites to be $n+1$. We now ask if there is a feasible solution of cost no more than $n s+n+s$. Note that if there exists a set cover of size $s$, then there exists a solution of cost $n s+n+s$. The reverse also holds, and therefore this completes the reduction.

Definition 6.1 An approximation algorithm for load balanced facility location is a $(\alpha, \beta)$ approximation for some $\alpha \geq 1$ and $\beta \leq 1$ if the cost of the solution is within $\alpha$ times the optimal cost and facility $i$, if opened, serves at least $L_{i} \beta$ demand.

Let us denote by $\rho_{f}$ the best known approximation ratio for classical facility location, which is $\rho_{f}=1.52$ due to [18]. We present a $\left(2 \rho_{f}, 1 / 3\right)$ approximation to this problem. The same result was independently obtained by [16]. Unlike classical facility location [22], the lower bound makes it hard to round the linear relaxation directly. This arises from the fact that the filtering steps of Lin and Vitter in [17] do not work. Thus fractional solutions cannot be rounded by previous approaches.

The Algorithm: The algorithm proceeds in two basic steps and uses an approximation algorithm for the facility location problem. We note that the approximation guarantee holds relative to the LP relaxation as well (albeit with more technical details that we omit, since it is not the main focus of the paper).

\section{Load Balanced Facility Location Algorithm}

(i) Transformation: For facility $i$, add the cheapest way to route exactly $L_{i}$ units of demand to $i$ to the facility cost $\phi_{i}$. To do this, consider demands in increasing order of distance from $i$, and route these demands to $i$ until exactly $L_{i}$ units have been routed. The routing cost of this process is added to $\phi_{i}$.

(ii): Facility Location: Next solve regular facility location with these facility costs using the $\rho_{f}$-approximation algorithm.

(iii) Rounding to Remove Facilities: Now consider the open facilities in arbitrary order. Consider any open facility $i$ that serves less than $L_{i} / 3$ amount of demand. Close the facility and route the demands it serves to their closest open facilities.

Lemma 6.1 Consider any feasible solution to the load balanced facility location problem of cost $C$. After the transformation in Step (i), this yields a feasible instance of the regular facility location problem of cost at most $2 C$.

Proof: Consider any facility $i$ opened by the load balanced solution. Since this solution is routing at least $L_{i}$ amount of demand to any open facility, the facility cost we assign in the new problem is at most the routing cost of the demand connected to that facility. Thus the total additional facility cost is at most $C$.

Therefore the total cost in the solution we compute is bounded in terms of the cost of the original solution to within a factor of $2 \rho_{f}$. Also note that facility location guarantees that each demand point goes to the closest open facility. We have to show that removing a facility does not increase the total facility plus routing cost of the solution. For this, we show a feasible way to route the demands it serves so that the cost does not increase.

Lemma 6.2 Removing a facility $i$ serving less than $\frac{L_{i}}{3}$ amount of demand cannot increase the cost of our solution. 
Proof: Suppose we are closing facility $i$. Consider the closest demand point $j$ which does not send demand to this facility. Suppose $c_{i j}=D$. If $j$ is being served by $i^{\prime}, c_{i^{\prime} j}<D$, as each demand point goes to the closest open facility.

Note that at least $2 L_{i} / 3$ units of demand are at distance $D$ or greater. Therefore, $f_{i}^{\prime} \geq \frac{2 L_{i}}{3} D$.

When we close the facility, we can afford to use $f_{i}^{\prime}$ towards re-routing the demand it serves. We send the demand to $i^{\prime}$, the facility serving $j$. The extra cost for doing this is at most the cost of taking the demand from $i$ to $j$ and from there to $i^{\prime}$. This distance is at most $2 D$ by the metric property, and the demand is at most $\frac{L_{i}}{3}$, and so the total re-routing cost is at most $\frac{2 L_{i}}{3} D$.

The above can be summarized in the following theorem:

Theorem 6.2 The load balanced facility location problem has a $\left(2 \rho_{f}, 1 / 3\right)$ approximation where each demand is served by its closest open facility.

\section{Conclusion}

In this paper, we presented the first constant factor approximation for the single-sink buy-at-bulk network design problem. We conclude by surveying the results on this and related problems that have appeared since the publication of the preliminary version of this paper [13]. First, the algorithm itself has been significantly improved and simplified. Gupta, Kumar, and Roughgarden [15] obtain a 72.8 approximation by combining the Steiner and shortest path stages into a "rent-or-buy" stage, and using a novel analysis. This is the current best known approximation guarantee. For the single-sink case, Goel and Estrin [11] consider simultaneous (oblivious) approximation over all concave functions, and obtain a $O(\log n)$ approximation.

For the multiple sink (source-sink pairs) version of this problem, as mentioned earlier, the best known approximation ratio of $O(\log n)$ follows directly from tree embeddings [3, 9]. For this version, Andrews [1] has shown a $\Omega\left((\log n)^{\frac{1}{4}}\right)$ hardness of approximation assuming $N P \not \subset \operatorname{DTIME}\left(n^{\log O(1)} n\right)$.

The non-uniform version of the problem assumes different cable types are available on different edges. As mentioned earlier, the best known approximation ratio [19] for the single-sink version is $O(\log n)$. Chuzhoy et al. [8] show that the single sink version is hard to approximate within $\Omega(\log \log n)$, under similar hardness assumptions as the uniform case. Charikar and Karagiozova [6] consider the non-uniform version in the presence of multiple source-sink pairs. The best result for this case is a polylogarithmic approximation ratio, and is achieved by Chekuri et al. [7].

\section{Acknowledgments}

We thank Matthew Andrews, Chandra Chekuri, and Serge Plotkin for several helpful discussions. This work was done while the authors were at Stanford University. Sudipto Guha was supported by an IBM Research Fellowship, NSF Grant IIS-9811904 and NSF Award CCR-9357849, with matching funds from IBM, Mitsubishi, Schlumberger Foundation, Shell Foundation, and Xerox Corporation. Adam Meyerson and Kamesh Munagala were supported by ARO grant DAAG55-98-1-0170 and ONR grant N00014-98-1-0589.

\section{References}

[1] M. Andrews. Hardness of buy-at-bulk network design. In Proceedings of the 45th Annual Symposium on Foundations of Computer Science, pages 115-124, 2004. 
[2] M. Andrews and L. Zhang. The access network design problem. 39th IEEE Symposium on Foundations of Computer Science, pages 40-49, 1998.

[3] B. Awerbuch and Y. Azar. Buy-at-bulk network design. Proceedings of the 38th IEEE Symposium on Foundations of Computer Science, pages 542-47, 1997.

[4] Y. Bartal. On approximating arbitrary metrics by tree metrics. 30th ACM Symposium on Theory of Computing, 1998.

[5] M. Charikar, C. Chekuri, A. Goel, S. Guha, and S. Plotkin. Approximating a finite metric by a small number of tree metrics. 39th IEEE Symposium on Foundations of Computer Science, 1998.

[6] M. Charikar and A. Karagiozova. On non-uniform multicommodity buy-at-bulk network design. Proceedings of STOC, pages 176-182, 2005.

[7] C. Chekuri, M. T. Hajiaghayi, G. Kortsarz, and M. R. Salavatipour. Approximation algorithms for non-uniform buy-at-bulk network design. Proceedings of the 47 th Annual Symposium on Foundations of Computer Science, pages 677-686, 2006.

[8] J. Chuzhoy, A. Gupta, J. Naor, and A. Sinha. On the approximability of network design problems. In Proceedings of the sixteenth annual ACM-SIAM symposium on Discrete algorithms, 2005 .

[9] J. Fakcharoenphol, S. Rao, and K. Talwar. A tight bound on approximating arbitrary metrics by tree metrics. In Proceedings of the thirty-fifth annual ACM symposium on Theory of computing, pages 448-455, 2003.

[10] N. Garg, R. Khandekar, G. Konjevod, R. Ravi, F. S. Salman, and A. Sinha. On the integrality gap of a natural formulation of the single-sink buy-at-bulk network design problem. Integer Programming and Combinatorial Optimization, 2001.

[11] A. Goel and D. Estrin. Simultaneous optimization for concave costs: single sink aggregation or single source buy-at-bulk. Proceedings of SODA, pages 499-505, 2003.

[12] S. Guha, A. Meyerson, and K. Munagala. Hierarchical placement and network design problems. Proceedings of 41 st IEEE FOCS, 2000.

[13] S. Guha, A. Meyerson, and K. Munagala. A constant factor approximation for the single sink edge installation problems. In Proceedings of the thirty-third annual ACM symposium on Theory of computing, pages 383-388, 2001.

[14] S. Guha and K. Munagala. Generalized clustering. Proceedings of SODA, page 484, 2002.

[15] A. Gupta, A. Kumar, and T. Roughgarden. Simpler and better approximation algorithms for network design. In Proceedings of the thirty-fifth annual ACM symposium on Theory of computing, pages 365-372, 2003.

[16] D. Karger and M. Minkoff. Building steiner trees with incomplete global knowledge. Proceedings of 41st IEEE FOCS, 2000.

[17] J.-H. Lin and J. S. Vitter. $\epsilon$-approximations with minimum packing constraint violations. Proceedings of the Twenty-Fourth Annual ACM Symposium on Theory of Computing, 1992.

[18] M. Mahdian, Y. Ye, and J. Zhang. Improved approximation algorithms for metric facility location problems. In Proceedings of the 5th International Workshop on Approximation Algorithms for Combinatorial Optimization, pages 229-242, 2002. 
[19] A. Meyerson, K. Munagala, and S. Plotkin. Cost-distance: Two metric network design. In Proceedings of the 41st Annual Symposium on Foundations of Computer Science, 2000.

[20] G. Robins and A. Zelikovsky. Improved steiner tree approximation in graphs. In Proc. $11^{\text {th }}$ Annual ACM-SIAM Symposium on Discrete Algorithms (SODA), pages 770-779, 2000.

[21] F. S. Salman, J. Cheriyan, R. Ravi, and S. Subramanian. Buy-at-bulk network design: Approximating the single-sink edge installation problem. Proceedings of the Eighth Annual ACMSIAM Symposium on Discrete Algorithms, pages 619-628, 1997.

[22] D. B. Shmoys, É. Tardos, and K. Aardal. Approximation algorithms for facility location problems. Proceedings of the Twenty-Ninth Annual ACM Symposium on Theory of Computing, pages 265-274, 1997.

[23] K. Talwar. The single-sink buy-at-bulk LP has constant integrality gap. Proceedings of IPCO, pages $475-486,2002$. 